\title{
Recirculación en rinosinusitis maxilar
}

\section{Mucus recirculation in maxillary rhinosinusitis}

\author{
Claudia Heider C1, Gloria Ribalta L², Karin Krauss M
}

\begin{abstract}
RESUMEN
Introducción: La recirculación de moco entre el ostium natural del seno maxilar y otras aberturas en el seno, altera el transporte mucociliar normal, favorece su acumulación y sobreinfección, perpetuando el fenómeno inflamatorio.

Objetivo: Describir nuestra experiencia en el diagnóstico y tratamiento de pacientes con rinosinusitis maxilar crónica y/o aguda recurrente cuyo estudio reveló recirculación maxilar.

Material y método: Estudio retrospectivo descriptivo. Se revisaron historias clínicas, nasofibroscopías, informes radiológicos y hallazgos operatorios.

Resultados: Ocho pacientes con edad promedio de 43 años. El 100\% presentó antecedente de cirugía que involucraba el seno maxilar. Todos se presentaron como rinosinusitis maxilar crónica y/o aguda con reagudizaciones frecuentes (3 episodios/ año). El diagnóstico se realizó mediante nasofibroscopía que evidenció recirculación de moco entre ostium natural y accesorio. Cinco pacientes se sometieron a cirugía endoscópica para unir el ostium accesorio (OA) al natural, disminuyendo el número de exacerbaciones.

Conclusión: La recirculación maxilar se debe sospechar en pacientes con diagnóstico de rinosinusitis maxilar crónica y/o aguda recurrente con antecedente de cirugías que involucren el seno maxilar. Su diagnóstico se basa en la observación de recirculación de moco a la nasofibroscopía. El manejo es quirúrgico y dependerá de la ubicación del OA y de la permeabilidad del ostium natural.
\end{abstract}

Palabras clave: Sinusitis maxilar, depuración mucociliar.

\begin{abstract}
Introduction: Recirculation of mucus between the maxillary sinus natural ostium and adjacent openings disturbs mucociliary clearence leading to secretions accumulation and persistent infection, perpetuating the inflammatory phenomenon.

Aim: Describe our experience in the diagnosis and treatment of patients with maxillary rhinosinusitis (chronic or acute recurrent) and maxillary recirculation.

Material and method: Descriptive and retrospective study. Clinical records, radiologic characteristic, nasal endoscopies and surgical findings were reviewed.

Results: 8 patients, mean age of 43 years. All of them had prior history of maxillary sinus surgery. Clinical presentation was maxillary rhinosinusitis, chronic or acute, with
\end{abstract}

Médico General.

2 Médico Otorrinolaringólogo, Clínica Las Condes. 
frequent episodes of reagudization (3 episodes/year). The diagnosis was made by endoscopic nasal examination that showed mucus recirculation between natural and accessory ostium. Endoscopic sinus surgery was done in 5 patients in order to communicate the accessory to the natural ostium, leading to a decrease in the number of exacerbations.

Conclusions: Maxillary recirculation must be suspected in patients with maxillary rhinosinusitis (chronic or acute recurrent) who have had prior surgical procedures that involve the maxillary sinus. The diagnosis is based on the observation of the recirculation phenomenon in the nasal endoscopy. The treatment is surgical and depends on the location of the accessory ostium and the permeability of the natural ostium.

Key words: Maxillary sinusitis, mucociliary clearance.

\section{INTRODUCCIÓN}

El fenómeno de recirculación en el seno maxilar involucra la circulación de moco entre el ostium natural del seno y otras aberturas ya sean "naturales" 0 iatrogénicas. Se describe la presencia de ostium accesorios (OA) en el seno maxilar en alrededor del $20 \%$ de los pacientes con rinosinutis crónica (RSC) afectando principalmente a la fontanela posterior de la pared lateral nasal. Se evidencia recirculación hasta en el $9 \%$ de ellos ${ }^{1}$. Su incidencia en pacientes sometidos previamente a cirugías nasosinusales es aún mayor, aunque desconocida, especialmente cuando la antrostomía queda separada del ostium natural del seno maxilar (SM). De esta manera, se ha reportado que la unión de ambos orificios removiendo el puente de tejido que las separa soluciona el problema.

Este fenómeno ha sido asociado a sinusitis recurrente y a fracaso de tratamiento quirúrgico. Su presencia se relaciona con infección crónica y algunos autores consideran que debiesen ser considerados un análogo a las perforaciones timpánicas en otitis media crónica denominando esta entidad el "síndrome de los 2 agujeros"1.

La presencia de $0 \mathrm{~A}$ puede cambiar radicalmente la ventilación del seno y afectar de manera adversa la función sinusal, el drenaje mucoso y la susceptibilidad a infecciones. En estudios computacionales de simulación de dinámica de fluidos en cavidades paranasales, Zhu estudió el efecto de $\mathrm{OA}$ en la ventilación sinusal y observó que la presencia de éstos aumentan considerablemente las tasas de flujo aéreo sinusal y alteran los patrones de circulación normal afectando de esta manera la concentración de óxido nítrico, la vulnerabilidad a bacterias, el clearence mucociliar y la llegada de drogas².
Sabemos que en individuos sanos, el drenaje mucoso normal del SM es hacia el ostium natural, ubicado en la parte más alta de la pared medial del seno, para luego ser dirigido a la nasofaringe. En individuos con $\mathrm{OA}$ este mecanismo se altera y el moco tiende a reentrar al SM por este agujero, dando origen al fenómeno de recirculación o "aniIlo mucoso". Este moco es más espeso y viscoso, y contiene una alta concentración de alérgenos, bacterias y mediadores inflamatorios aumentando así el fenómeno de inflamación crónica sinusal ${ }^{3}$.

\section{OBJETIVO}

El objetivo de este trabajo es describir la experiencia en el diagnóstico y tratamiento de una serie de 8 pacientes atendidos en Clínica Las Condes con diagnóstico de rinosinusitis maxilar crónica y/o aguda recurrente cuyo estudio reveló la presencia de $0 A$ y recirculación maxilar.

\section{MATERIAL Y MÉTODO}

Estudio descriptivo retrospectivo de una serie de casos incidentes. Se estudiaron pacientes atendidos en el Departamento de Otorrinolaringología de Clínica Las Condes entre 2005 y 2012 que presentaban rinosinusitis maxilar crónica y/o aguda recurrente cuyo estudio reveló fenómeno de recirculación.

Se utilizaron las siguientes definiciones:

- Ostium accesorio (OA): Cualquier abertura a nivel del seno maxilar (frecuentemente quirúrgica) que no corresponda a su ostium natural.

- Fenómeno de recirculación: Visualización de OA con salida de moco. 
Para el diagnóstico de Rinosinutis Aguda (RSA) Recurrente y Rinosinusitis Crónica (RSC) se utilizaron los criterios planteados por la Academia Americana de Otorrinolaringología y Cirugía de Cabeza y Cuello. (Tabla 1$)^{4}$.

Se registraron características biodemográficas, antecedentes quirúrgicos, presentación clínica de la enfermedad, estudio de imágenes, hallazgos nasofibroscópicos y quirúrgicos y evolución clínica. Se utilizó estadística descriptiva. (Medidas de tendencia central y rangos).

\section{RESULTADOS}

Se estudiaron un total de 8 pacientes: 5 mujeres y 3 hombres con una edad promedio de 43 años $\pm 11,6$ (28 a 63 años). El 100\% de ellos presentaba el antecedente de cirugía previa a nivel del seno maxilar (Tabla 2). Cuatro pacientes se presentaron como RSC maxilar con reagudizaciones frecuentes y cuatro pacientes como RSA maxilar recurrente. El promedio de episodios fue de $3 \pm 1,1$ episodios por año.

Tabla 1. Criterios diagnósticos para rinosinusitis crónica y rinosinusitis aguda recurrente

Rinosinusitis crónica (RSC): 12 o más semanas de 20 más de los siguientes síntomas y signos:

- $\quad$ Descarga mucopurulenta (anterior, posterior 0 ambas)

- Obstrucción nasal (congestión)

- $\quad$ Algia o plenitud facial 0

- Hiposmia

Y documentación de la inflamación a través de uno o más de los siguientes hallazgos:

- $\quad$ Mucosidad purulenta o edema en meato medio o región etmoidal

- $\quad$ Pólipos en cavidad nasal o meato medio y/o

- Imágenes radiológicas que muestren inflamación de cavidades paranasales

Rinosinusitis aguda recurrente: Cuatro (4) o más episodios de rinosinusitis aguda (RSA) al año sin síntomas o signos entre cada episodio.

- Cada episodio de RSA debe cumplir criterios diagnósticos (Guía Academia Americana de Otorrinolaringología y Cirugía de Cabeza y Cuello)

Tabla 2. Descripción de los casos

\begin{tabular}{|c|c|c|c|c|c|c|}
\hline Paciente & Edad & Género & $\begin{array}{l}\text { Antecedente } \\
\text { quirúrgico }\end{array}$ & Diagnóstico & Ubicación $0 \mathrm{~A}$ & Tratamiento \\
\hline 1 & 57 & $M$ & $\begin{array}{l}\text { Avance tercio } \\
\text { medio facial }\end{array}$ & RSAr SMD & $\begin{array}{l}\text { Meato inferior } \\
\text { bilateral } \\
\text { (osteotomía } \\
\text { de avance) }\end{array}$ & $\begin{array}{l}\text { En espera de } \\
\text { cierre } \\
\text { espontáneo }\end{array}$ \\
\hline 2 & 46 & M & CEFS & RSC SMI & Meato medio SMI & CEFS \\
\hline 3 & 34 & $\mathrm{H}$ & CEFS & RSC SMI & $\begin{array}{l}\text { Meato medio } \\
\text { bilateral }\end{array}$ & $\begin{array}{l}\text { En espera de } \\
\text { CEFS }\end{array}$ \\
\hline 4 & 39 & M & $\begin{array}{l}\text { Meatotomía } \\
\text { inferior }\end{array}$ & $\begin{array}{l}\text { RSC maxilar } \\
\text { bilateral }\end{array}$ & $\begin{array}{l}\text { Meato inferior } \\
\text { bilateral }\end{array}$ & CEFS \\
\hline 5 & 40 & M & CEFS & RSAr SMD & Meato medio SMD & CEFS \\
\hline 6 & 39 & M & CEFS & RSAr SMD & Meato medio SMD & CEFS \\
\hline 7 & 63 & $\mathrm{H}$ & $\begin{array}{l}\text { CEFS } \\
\text { bilateral }\end{array}$ & $\begin{array}{l}\text { RSC maxilar } \\
\text { bilateral }\end{array}$ & Meato medio & CEFS \\
\hline 8 & 28 & $\mathrm{H}$ & $\begin{array}{l}\text { CEFS } \\
\text { bilateral }\end{array}$ & $\begin{array}{l}\text { RSAr maxilar } \\
\text { bilateral }\end{array}$ & Meato medio & CEFS \\
\hline
\end{tabular}

H: Hombre; M: Mujer; RSC: Rinosinusitis Crónica; RSAr: Rinosinusitis aguda recurrente; CEFS: Cirugía endoscópica funcional de senos; SMD: Seno maxilar derecho; SMI: Seno maxilar izquierdo. 
El diagnóstico se hizo en siete pacientes $(87,5 \%)$ en base a la historia clínica asociado a antecedente de cirugía a nivel de seno maxilar, tomografía computarizada de cavidades perinasales que demostraba presencia de OA y nasofibroscopía que evidenciaba salida de moco a nivel del $O A$ (Figura 1). En un paciente este fenómeno fue un hallazgo intraoperatorio. El tiempo transcurrido entre la cirugía y el diagnóstico de recirculación fue de $9 \pm 6,1$ años (2 a 20 años).

Tres pacientes fueron evaluados por inmunología, diagnosticándose en un paciente una inmunodeficiencia humoral secundaria a macroglobulinemia de Waldenstrom.

La ubicación del OA fue en 6 pacientes a nivel del meato medio. Todos ellos presentaban antecedente de cirugía funcional endoscópica de senos (CEFS) practicada previamente en nuestro centro y en otros. Los otros 2 pacientes presentaron $\mathrm{OA}$ a nivel de meato inferior: uno con antecedente de meatotomía inferior secundaria a cirugía de Caldwell-Luc y el otro con antecedente de osteotomía de avance del tercio medio facial que dejó una solución de continuidad en la pared medial ósea del meato inferior, por donde salía moco.

Seis de los 8 pacientes han recibido a la fecha tratamiento quirúrgico. Se realizó diferente tipo de cirugía según la ubicación del OA: En aquellos cinco pacientes con $0 \mathrm{~A}$ a nivel de meato medio se procedió a unir el ostium accesorio al natural mediante una antrostomía amplia (Figura 2). En los 2 casos en los cuales el OA estaba en meato inferior, el tratamiento quirúrgico dependió de la permeabilidad del infundíbulo maxilar; si éste estaba obstruido, como fue el caso del paciente con antecedente de cirugía previa de Caldwell-Luc, se

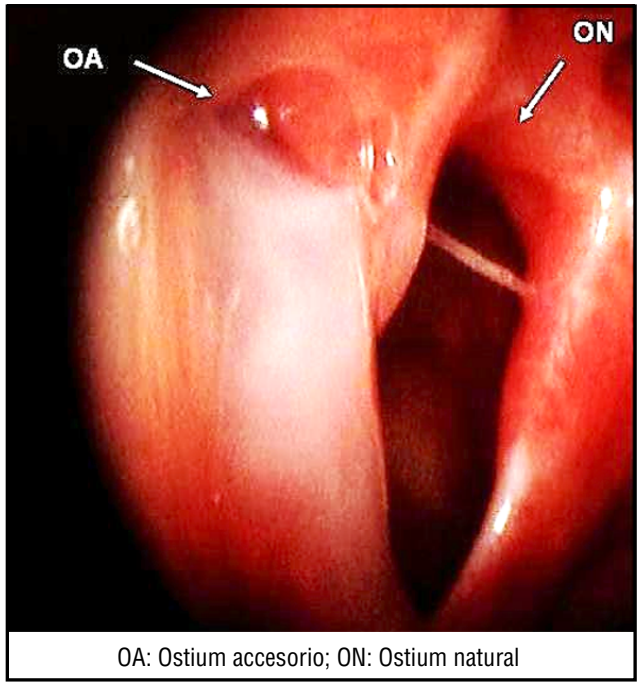

Figura 1. Fenómeno de recirculación maxilar. Caso 5: Se observa salida de moco desde el ostium accesorio hacia antrostomía previa maxilar derecha, dando origen al fenómeno de recirculación.

procedió a hacer una antrostomía maxilar media para facilitar el drenaje natural del moco y dejar que cerrara en forma espontánea el ostium accesorio inferior. En el otro caso en el cual el ostium accesorio fue secundario a osteotomía de avance, el infundíbulo maxilar estaba permeable, por lo cual se está esperando la evolución espontánea, permitiendo el cierre del OA en forma natural.

El tiempo promedio de seguimiento desde la cirugía, en los 6 pacientes intervenidos, ha sido de 18 meses ( 1 a 44 meses). Durante este periodo se han detectado en promedio una reagudización de rinosinusitis maxilar, salvo en el paciente portador de macroglobulinemia de Waldenstrom, que ha presentado un mayor número de reagudizaciones.

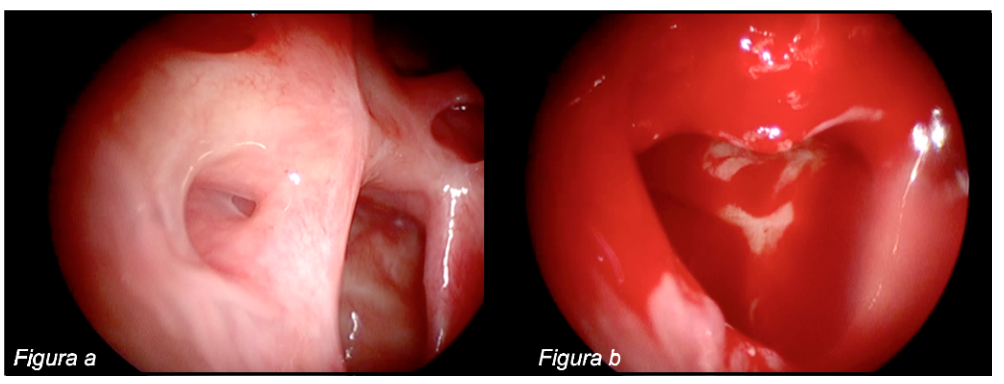

Figura 2. Caso 5: Imagen quirúrgica con óptica de $45^{\circ}$ que demuestra en a: presencia de ostium accesorio y $b$ : ambos ostium unidos luego de resecar el puente mucoso entre ambos. 


\section{DISCUSIÓN}

El fenómeno de recirculación de moco entre aperturas adyacentes hacia el ostium natural del seno maxilar es una situación que se da con relativa frecuencia en pacientes sometidos previamente a cirugías a nivel del seno maxilar, correspondiendo en nuestra serie al $100 \%$ de los pacientes, siendo más frecuente en aquellos sometidos a CEFS. Esta situación se podría deber al uso inadecuado de ópticas anguladas, quedando el ostium natural fuera de la visión directa del cirujano y de esta manera, la antrostomía se crea en la fontanela posterior, separada del ostium natural, favoreciendo el fenómeno de recirculación de moco5 (Figura 3).

Fisiopatológicamente la presencia de estos $\mathrm{OA}$ afectaría la ventilación sinusal y a su vez el clearence mucociliar, provocando ectasia de las secreciones con una mayor susceptibilidad a sobreinfecciones bacterianas y a fenómenos de inflamación crónica, perpetuando y provocando reagudizaciones frecuentes de $\mathrm{RSC}$, explicando a su vez la presentación clínica de estos pacientes.

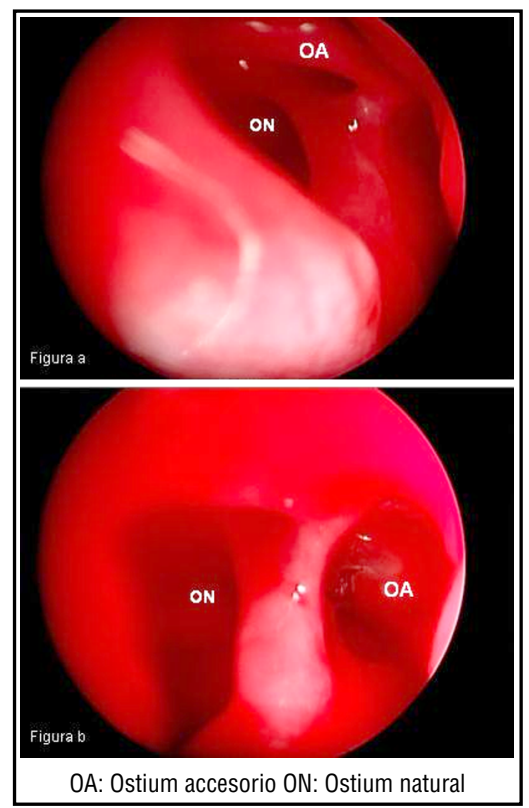

Figura 3. a): Visualización con óptica de $0^{\circ}$. b): Visualización con óptica de $45^{\circ}$. Al comparar ambas figuras se observa claramente como una óptica de $45^{\circ}$ brinda una mejor imagen cuando debemos trabajar en la pared medial del seno maxilar a nivel del ostium natural.
El diagnóstico se realiza mediante nasofibroscopía permitiendo visualizar la presencia de OA, su ubicación, el movimiento de mucus a través de ellos e incluso la presencia de anillo mucoso $0^{3,6}$. La presencia de moco puede variar en el tiempo, siendo necesario repetir el examen en ocasiones para confirmar el diagnóstic $0^{5}$.

La tomografía computarizada de cavidades perinasales puede ser un elemento útil en el diagnóstico de la recirculación y permite visualizar el anillo mucoso en los cortes coronales siendo difícil de evidenciar cuando existe edema de las estructuras vecinas ${ }^{7}$. Todos los pacientes de nuestra serie fueron evaluados con TC de cavidades perinasales que evidenció la presencia de $0 \mathrm{~A}$ pero, al contrario de lo reportado en la literatura, no fue posible visualizar la presencia de moco en forma de "anillo mucoso".

En relación al tratamiento, en base a estudios observacionales la literatura propone ante la existencia de $\mathrm{OA}$ a nivel de meato medio, la unión de ambos orificios, ostium natural y $O A$ en una sola gran antrostomía, situación que desde el punto de vista fisiopatológico permitiría solucionar el problema. No existen en la literatura estudios de terapia que evalúen la efectividad de este tratamiento. A todos nuestros pacientes con $\mathrm{OA}$ a nivel de meato medio se les planteó la posibilidad de tratamiento quirurgico para unir ambos orificios. En ellos, la evolución a 18 meses promedio ha sido muy favorable, con disminución del número de reagudizaciones.

En los pacientes en que el fenómeno de recirculación ocurre a nivel de la antrostomía quirúrgica previa del meato inferior, situación menos frecuente y secundaria a cirugías menos habituales, el tratamiento es diferente. Se debe abrir el ostium natural si éste no fue abierto en la cirugía previa y se encuentra obstruido, y así, al favorecer el drenaje natural por el ostium natural ampliado, esperar el cierre espontáneo del OA. Si el ostium natural está permeable se puede realizar un seguimiento nasofibroscópico y esperar el cierre natural del $O A$, siempre que no exista cronificación del proceso inflamatorio y que la tendencia natural sea a la disminución del número de exarcerbaciones. Aun cuando el número de casos presentados es bajo, consideramos que nuestra serie aporta $y$ enfatiza un concepto fisiológico y fisiopatológico 
básico. La presencia de $0 \mathrm{~A}$ y recirculación altera el transporte mucociliar normal y perpetúa el fenómeno inflamatorio, situación aún más frecuente en pacientes sometidos previamente a cirugías nasosinusales. Creemos entonces que razonablemente se podría plantear como hipótesis la recirculación maxilar como causa de rinosinutis crónica y/o aguda recurrente 0 como una causa de fracaso a tratamiento quirúrgico y diseñar un estudio apropiado que responda a esta pregunta. De la misma manera, el fenómeno de recirculación a nivel de otros senos (frontal, esfenoidal) podría jugar también un rol en la perpetuación de fenómenos inflamatorios y mala evolución clínica de los pacientes, siendo necesario más estudios al respecto.

\section{CONCLUSIONES}

Recomendamos sospechar y buscar dirigidamente el fenómeno de recirculación maxilar en pacientes con diagnóstico de RSA y/o crónica con reagudizaciones frecuentes y que hayan sido sometidos previamente a cirugías que involucren manipulación del seno maxilar, particularmente CEFS. Esta condición se diagnostica mediante nasofibroscopía y su manejo es quirúrgico y dependerá de la ubicación del $O A$ y de la permeabilidad del ostium natural. Cuando el $O A$ se encuentra a nivel del meato medio se deben unir ambos orificios en una sola antrostomía. Si el OA está en meato inferior y el ostium natural está obstruido, se debe abrir el ostium natural, para favorecer el drenaje natural del moco y esperar el cierre espontáneo del OA de meato inferior. Si el ostium natural está abierto, se puede esperar a un cierre natural del $O A$ de meato inferior, siempre y cuando no exista cronificación del proceso inflamatorio y que la tendencia natural sea a la disminución del número de exarcerbaciones.

\section{BIBLIOGRAFÍA}

1. Mladina R, Vukovic K, Poje G. The two holes syndrome. Am J Rhinol Allergy 2009; 23(6): 6024.

2. Zhu JH, Lee HP, Lim KM, Gordon BR, Wang de Y. Effect of accessory ostia on maxillary sinus ventilation: a computational fluid dynamics (CFD) study. Respir Physiol Neurobiol 2012; 15; 183(2): 91-9.

3. Matthews BL, Burke AJ. Recirculation of mucus via accessory ostia causing chronic maxillary sinus disease. Otolaryngol Head Neck Surg 1997; 117(4): 422-3.

4. Rosenfeld R, Andes D, Bhattacharyva N, et al. Clinical practice guideline. Adult sinusitis. Otolaryngol Head Neck Surg 2007; 137 Suppl: S1-S31.

5. Kane KJ. Recirculation of mucus as a cause of persistent sinusitis. Am J Rhinol 1997; 11(5): 361-9.

6. Chung SK, Dhong HJ, Na DG. Mucus circulation between accessory ostium and natural ostium of maxillary sinus. J Laryngol Otol 1999; 113(9): 865-7.

7. Chung SK, Cho DY, Dhong HJ. Computed tomogram findings of mucous recirculation between the natural and accessory ostia of the maxillary sinus. Am J Rhinol 2002; 16(5): 265-8. 